\title{
Candidate gene polymorphisms for diabetes mellitus, cardiovascular disease and cancer are associated with longevity in Koreans
}

\author{
Ji Wan Park ${ }^{1 *}$, Yong Ick $\mathrm{Ji}^{2 *}$, Yoon-Ho Choi ${ }^{3}$, \\ Mi-Yeon Kang ${ }^{2}$, Eunhyun Jung ${ }^{2}$, Se Young $\mathrm{Cho}^{2}$, \\ Hee-Youn $\mathrm{Cho}^{2}$, Byung Kyu Kang ${ }^{2}$, \\ Yoo Sook Joung ${ }^{4}$, Duk-Hwan Kim ${ }^{2,5}$ \\ Sang Chul Park ${ }^{6,7}$ and Joobae Park ${ }^{2,5,7}$ \\ ${ }^{1}$ Department of Medical Genetics \\ Hallym University College of Medicine \\ Chuncheon 200-702, Korea \\ ${ }^{2}$ Center for Genome Research \\ Samsung Biomedical Research Institute \\ ${ }^{3}$ Department of Internal Medicine \\ ${ }^{4}$ Department of Psychiatry \\ ${ }^{5}$ Department of Molecular Cell Biology \\ Sungkyunkwan University School of Medicine \\ Seoul 135-710, Korea \\ ${ }^{6}$ Aging and Apoptosis Research Center \\ Seoul National University Institute on Aging \\ Seoul 110-799, Korea \\ ${ }^{7}$ Corresponding authors: Tel, 82-2-3410-3600; \\ Fax, 82-2-3410-3620; E-mail, jbpark@med.skku.ac.kr (J.B. Park), \\ Tel, 82-2-740-8244; Fax, 82-2-744-4534; \\ E-mail, scpark@snu.ac.kr (S.C. Park) \\ *These authors contributed equally to this work. \\ DOI 10.3858/emm.2009.41.11.083
}

Accepted 22 June 2009

Abbreviations: $A B C C 8$, ATP-binding cassette, sub-family C (CFTR/ MRP), member 8; $A C E$, angiotensin I converting enzyme (peptidyldipeptidase A) 1; $A D C Y 2$, adenylate cyclase 2 (brain); $A L D H 2$, aldehyde dehydrogenase 2 family (mitochondrial); $A P O E$, apolipoprotein E; CDK10, cyclin-dependent kinase 10; CETP, cholesteryl ester transfer protein, plasma; $\mathrm{Cl}$, confidence interval; $\mathrm{CR}$, call rate; CVD, cardiovascular disease; DM, diabetes mellitus; HWE, Hardy-Weinberg equilibrium; IL12RB2, interleukin 12 receptor, beta 2; LD, linkage disequilibrium; LRM, logistic regression model; $L Y N$, V-yes-1 Yamaguchi sarcoma viral related oncogene homolog; MAF, minor allele frequency; MGP, matrix Gla protein; MTTP, microsomal triglyceride transfer protein; $\mathrm{OR}$, odds ratio; $P A X 4$, paired box 4; $P C S K 1$, convertase subtilisin/kexin type 1; PON1, paraoxonase 1; PPP1R14C, protein phosphatase 1, regulatory (inhibitor) subunit 14C; PPP1R1A, protein phosphatase 1, regulatory (inhibitor) subunit $1 \mathrm{~A}$; SNP, single nucleotide polymorphism; TNFSF11, tumor necrosis factor (ligand) superfamily, member 11

\begin{abstract}
Long-lived people may have a unique genetic makeup that makes them more resistant than the general population to prevalent age-related diseases; however, not much is known about genes involved in the longevity. To identify susceptibility variants controlling longevity, we performed a high-throughput candidate gene study using 137 Koreans over $90 \mathrm{yr}$ old and 213 young healthy Koreans. We evaluated 463 informative markers located in 176 candidate genes mostly for diabetes mellitus, cardiovascular disease and cancer under five genetic models. We estimated the odds ratios for each allele, genotype, haplotype, and gene-gene interaction using logistic regression analysis. Associations between $\mathbf{1 3}$ genes and longevity were detected at a $P$-value less than 0.01 . Particularly, the rs671 (A) allele of the aldehyde dehydrogenase 2 family (mitochondrial) (ALDH2) gene was associated with longevity only in men (OR 2.11, $P$ $=0.008$ ). Four genes, proprotein convertase subtilisin/kexin type 1 (PCSK1, $P=0.008)$, epidermal growth factor receptor (EGFR, $P=0.003)$, paired box $4(P A X 4, P=0.008)$, and V-yes-1 Yamaguchi sarcoma viral related oncogene homolog $(L Y N, P=0.002)$ consistently yielded statistical evidence for association with longevity. The findings of the current study may provide a starting point for future studies to unravel genetic factors controlling longevity in Koreans.
\end{abstract}

Keywords: case-control studies; genome- wide association study; longevity; polymorphism, single nucleotide

\section{Introduction}

In the past $50 \mathrm{yr}$, the mean life expectancy in most developed countries has increased from around 50 $\mathrm{yr}$ to $80 \mathrm{yr}$. This change suggests that environmental changes may influence on extending the human lifespan. At the same time, variation in maximum lifespan among species and genetic variations in lifespan across human populations clearly points toward a genetic basis for lifespan (Christensen et al., 2006; Bergman et al., 2007). 
According to a birth cohort study from north-east America, having a centenarian sibling increased the chance of survival beyond ninety years old by four times, indicating a strong familial aggregation to longevity (Perls et al., 1998). The heritability of lifespan was around $33 \%$ and did not vary by gender in Danish twins (McGue et al., 1993). Linkage study is often considered inappropriate for complex traits; however, a region of chromosome 4 was shown to be linked with exceptional longevity (Puca et al., 2001). Gene-environment interactions (e.g. ACE, angiotensin I converting enzyme (peptidyl-dipeptidase A) 1 and exercise; IL6, interleukin 6 (interferon, beta 2) and zinc) also contribute to the development of aging-related phenotypes (e.g. physical decline and inflammation, respectively) (Capri et al., 2006).

The frequency of centenarians is approximately $1 / 100,000$ people worldwide. According to the Korea National Statistical Office (KNSO) Report in 2005 (http://www.kosis.kr.), the frequencies of nonagenarians and centenarians were 131 and 2/100,000 Koreans, respectively (KNSO, 2005). Centenarians may not be free of disease, but manage to survive with or without treatment owing to their resistance to disease (Franceschi et al., 2000). The incidences of major common diseases slow down or even decline around age 85-90 yr (Bergman. et al., 2007). Gender also accounts for important differences in longevity as well as in the occurrence of a variety of age-related disease. The male/female ratios were about 1:4 in nonagenarians and 1:8.2 in centenarians in Korea (KNSO, 2005). Such data raise the possibility of gender-specific genetic determinants of human longevity.

Approximately 30 candidate genes for longevity have been reported in previous studies since Herman (1956) proposed the free radical theory of aging, where oxidative stress causes aging (Salvioli et al., 2006). Genes involved in inflammation and the immune response (e.g. IL6; LMP2, low molecular mass protein 2; TNFA, tumor necrosis factor (TNF superfamily, member 2); TGFB1, transforming growth factor, beta 1 ; and PPAR $\gamma$, peroxisome proliferator-activated receptor gamma), genome maintenance and repair (e.g. P53), lipid metabolism (e.g. APOE, apolipoprotein E; CETP, cholesteryl ester transfer protein, plasma; MTTP, microsomal triglyceride transfer protein, and PON1, paraoxonase 1), glucose metabolism (e.g. IGF1, insulin-like growth factor 1 (somatomedin $C$ ); and HFE, hemochromatosis), oxidative stress (e.g. SOD1, superoxide dismutase 1 , soluble; PARP, poly (ADP-ribose) polymerase 1; and GSTT1, glutathione S-transferase theta 1), mitochondrial mutation, premature aging syndromes such as Werner syndrome (i.e. WRN), and telomere length have been considered with respect to both age-related diseases and longevity (Atzmon et al., 2006; Capri et al., 2006; Christensen et al., 2006; Salvioli et al., 2006).

Genes involved in increased lifespan may play protective roles in the development of age-related diseases. The presence of deleterious genotypes of age-related diseases may be a surrogate indicator of aging. Genes associated with aging-related diseases often have pleiotropic effects, while epistatic interactions affect human longevity (Christensen et al., 2006). For instance, significant decreases of paraoxonase (i.e. PON1) activity on the surface of HDL have been observed in both diabetes mellitus (DM) and cardiovascular disease (CVD) (Mackness et al., 2004). Although association between DM and cancer remains controversial, an increased risk of lung cancer has been observed among diabetic Korean women (hazard ratio $1.39,95 \%$ confidence interval 1.10-1.76) (Jee et al., 2005) and such experimental evidence has suggested that both insulin and insulin-like growth factors (IGFs) can stimulate tumor cell proliferation (Rousseau et al., 2006).

Because the difficulty in finding centenarians or nonagenarians limits the replication of genome-wide association (GWA) studies using an extremely large number of randomly spaced markers, we adopted a high-throughput candidate gene approach to identify susceptibility variants controlling lifespan in Koreans.

\section{Results}

Among 565 polymorphic SNPs analyzed, 102 SNPs $(18 \%)$ with MAF $<2 \%$, HWE $P<10^{-4}$, or CR $<$ $95 \%$ in the young age group did not passed the threshold of our quality test for genotypes. Thus, a total of 463 informative markers located in 176 genes were included in the subsequent analyses. For the comparison of the individuals above age 90 versus the young healthy controls, our study had a sample size adequate to reach a power of $80 \%$ to significantly detect a genotypic OR over 1.7/2.4 (heterozygote/ homozygote) under the assumptions shown above (see the Methods). The result obtained from centenarian women was shown in Supplemental Data Tables.

\section{Allelic- and genotypic association}

A total of 43 SNPs located in 33 genes $(18.8 \%$ of 176 candidate genes) had a nominally significant association with lifespan $(P<0.05)$ in the allelic $\chi^{2}$ 
tests (data not shown). Among these, 34 SNPs of 28 genes showed a significant allelic OR and 7 genes had a $P$ value less than 0.01 in at least one age/gender group (i.e. IL12RB2, interleukin 12 receptor, beta 2; PPP1R14C, protein phosphatase 1 , regulatory (inhibitor) subunit 14C; EGFR, epidermal growth factor receptor; $P A X 4$, paired box 4 ; $M M P 1$, matrix metallopeptidase 1 (interstitial collagenase); PPP1R1A, protein phosphatase 1, regulatory (inhibitor) subunit $1 \mathrm{~A}$; and $A L D H 2$, aldehyde dehydrogenase 2 family (mitochondrial)). A total of 128 SNPs located in 72 genes ( $41 \%$ of 176 genes) were statistically associated with one or more age/gender groups $(P<0.05)$ using regression models for five modes of inheritance (data not shown). Among these, the genotypic ORs of 95 SNPs located in 68 genes were nominally significant in at least one of age/gender groups and 13 genes had a $P$ value less than 0.01 (Table 1). We present the $P$ values corrected by genomic control where the $\lambda$ is greater than 1 (i.e. female centenarians, log-additive model, $\lambda=1.05$ ). As shown in Table 1, the genotypic effect on longevity was stronger than the corresponding allelic effect in either direction (i.e. either increased or decreased lifespan).

A total of 27 SNPs located in 25 genes showed evidence for association with lifespan in both gender groups (data not shown). Although the allelic effects of the matrix Gla protein (MGP) and the tumor necrosis factor (ligand) superfamily, member 11 (TNFSF11) were insignificant, these genes showed notable genotypic effects in the overdominant model. As shown in Table 1, TG heterozygotes of the rs 1054016 were observed at lower frequencies among the old age group compared to the young age group (OR 0.45 and 0.32 and $0.30 ; P=4 \times 10^{-4}$ and 0.001 for each group of nonagenarians adjusted for gender and males $\geq 90$, respectively). All 27 genes consistently showed evidence for association with lifespan in both allele- and genotype-based analyses. In particular, six genes showing significant association with a $P$ value less than 0.01 ( $A D C Y 2$, adenylate cyclase 2 (brain), PPP1R1A; LYN, V-yes-1 Yamaguchi sarcoma viral related oncogene homolog; PAX4, ALDH2, IL12RB2) attracted considerable attention as candidate genes affecting lifespan in Koreans. The results revealed significant gender differences in genetic associations with human longevity.

\section{Nonagenarian men}

A total of 43 SNPs located in 31 genes including four missense mutations located in each of euka- ryotic translation initiation factor 4 gamma, 1 (EIF4G1), ATP-binding cassette, sub-family C (CFTR/MRP), member 8 (ABCC 8 ), ALDH2, and telomerase-associated protein 1 (TEP1) showed evidence for genotypic association and 9 genes yielded $P$ values less than 0.01 (Table 1B). By way of example, a male with an A allele of the rs671 (Lys504Glue) located in the $A L D H 2$ increased the chance of living longer than $90 \mathrm{yr}$ by 2.11 times compared to men having a $\mathrm{G}$ allele $(P=0.009)$ and this SNP fitted best under a dominant model (OR 2.63, $P=0.004$ ). No allelic effect of the rs757110 (Ser1369Ala) in the $A B C C 8$ gene was identified; however, the effect of the TG heterozygous genotype was greater than that of the rare GG homozygous type (OR $3.12, P=6 \times 10^{-4}$ ).

\section{Nonagenarian women}

A total of 37 SNPs located in 31 genes showed evidence for genotypic associations in either age group of women (i.e. women aged over $90 \mathrm{yr}$ and centenarian women). Genes such as PPP1R14C ( $P$ $=0.005)$ and the EGFR $(P=0.007)$ had lower allelic $P$ values in the centenarian group with a small sample size (data not shown), while IL12RB2 was significant only in the younger group with more subjects $(P=0.008)$. However, the rare T allele of rs2030071 was not sufficiently common to determine the genetic model (Table 1C).

\section{Haplotypic association}

Among the 69 genes that showed significant genotypic association, we found significant association with longevity in 12 genes by using tagSNPs for 32 genes with at least 3 genotyped SNPs. Among nine genes that consistently yielded significant evidence of allelic, genotypic, and haplotypic associations with longevity (i.e. HK2, hexokinase 2; $E R B B 4$, v-erb-a erythroblastic leukemia viral oncogene homolog 4 (avian); PCSK1, proprotein convertase subtilisin/kexin type 1; ITK, IL2-inducible T-cell kinase; EGFR; PAX4; LPL, lipoprotein lipase; $L Y N$; and CDK10, cyclin-dependent kinase 10), only the PCSK1 $(P=0.008)$, EGFR $(P=0.003)$, $P A X 4(P=0.008)$, and $L Y N(P=0.002)$ remained significant after the Bonferroni correction for multiple testing of haplotypes in each gene (Table 2). Figure 1 summarizes the $-\log _{10}(P$ value) obtained from three different analyses of association for these four genes.

The presence of the $G$ allele of rs 155979 in the CGA haplotype of PCSK1 decreased lifespan among the group of nonagenarian adjusted by sex (OR 0.54). The $G$ allele of rs712700 increased 
Table 1. Results from the allele-and genotype-based analyses for individual SNPs showing evidence for association with longevity $(P<$ 0.01).

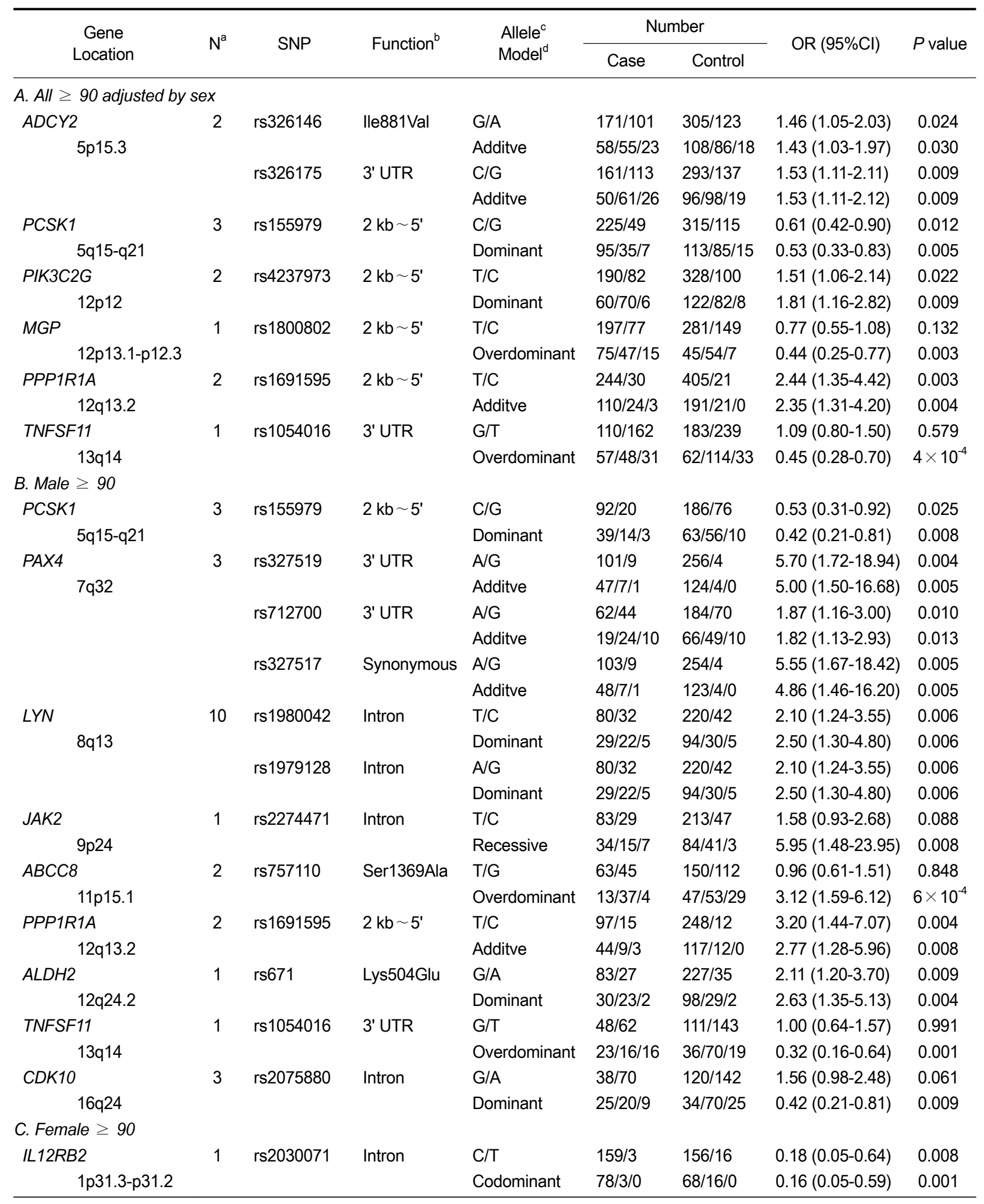

${ }^{a}$ Number of informative markers for the gene; ${ }^{b} U T R$, un-translated region; ${ }^{c}$ Ancestral alleles were shown in bold; ${ }^{d}$ Best fitting mode of inheritance: Only the SNPs showing a significant genotypic OR from the most significant regression model $(P<0.01)$ for the gene were shown. 
Table 2. Results from the haplotype-based analyses for candidate genes showing evidence for association with longevity $(P<$ $0.05 /$ Number of haplotypes).

\begin{tabular}{|c|c|c|c|c|c|c|}
\hline Gene & SNPs & $\begin{array}{l}\text { Group } \\
P \text { value }^{\mathrm{b}}\end{array}$ & Haplotypes & Freq & OR & $95 \% \mathrm{Cl}$ \\
\hline PCSK1 & rs3811952-rs155979 & Age $\geq 90$ & CCA & 0.302 & 1.00 & Reference \\
\hline \multirow[t]{5}{*}{$5 q 15-q 21$} & -rs3762985 (3/3) ${ }^{a}$ & 0.628 & $\mathrm{CCC}$ & 0.247 & 0.89 & $0.56-1.43$ \\
\hline & & 0.008 & CGA & 0.228 & 0.54 & $0.34-0.85$ \\
\hline & & 0.752 & GCA & 0.156 & 0.92 & $0.53-1.58$ \\
\hline & & 0.996 & GCC & 0.060 & 1.00 & $0.48-2.09$ \\
\hline & & 0.246 & Rare & 0.007 & 4.59 & $0.35-60.17$ \\
\hline EGFR & rs2072454-rs2293347- & Female $\geq 100$ & CACA & 0.310 & 1.00 & Reference \\
\hline \multirow[t]{6}{*}{$7 \mathrm{p} 12$} & rs3807362-rs884225 & 0.420 & TGCG & 0.247 & 0.67 & $0.25-1.77$ \\
\hline & $(4 / 4)$ & 0.003 & CGCA & 0.187 & 4.11 & $1.64-10.31$ \\
\hline & & 0.339 & CGCG & 0.150 & 1.60 & $0.61-4.18$ \\
\hline & & 0.000 & TACA & 0.052 & 0.00 & - \\
\hline & & 0.329 & TGCA & 0.033 & 0.31 & $0.03-3.27$ \\
\hline & & 0.000 & CATA & 0.022 & 0.00 & N.a. \\
\hline$P A X 4$ & rs327519-rs712700 & Male $\geq 90$ & $\mathrm{AA}$ & 0.683 & 1.00 & Reference \\
\hline \multirow[t]{2}{*}{$7 q 32$} & $(2 / 3)$ & 0.130 & $A G$ & 0.282 & 1.49 & $0.89-2.51$ \\
\hline & & 0.008 & GG & 0.035 & 5.14 & $1.54-17.14$ \\
\hline$L Y N$ & rs1450740-rs1450741- & Age $\geq 90$ & CTCTGCA & 0.504 & 1.00 & Reference \\
\hline \multirow[t]{21}{*}{$8 q 13$} & rs1562818- rs1980042- & 0.141 & ATCCGCA & 0.119 & 1.46 & $0.88-2.41$ \\
\hline & rs1027989-rs1546518- & 0.741 & CTTTGCA & 0.114 & 1.10 & $0.64-1.89$ \\
\hline & rs3968371 & 0.325 & CСCTGCA & 0.077 & 1.37 & $0.73-2.58$ \\
\hline & $(7 / 10)$ & 0.155 & ATCTGCA & 0.052 & 1.77 & $0.81-3.88$ \\
\hline & & 0.002 & CTCCGCA & 0.048 & 3.72 & $1.64-8.45$ \\
\hline & & 0.900 & CTCCGGG & 0.037 & 0.95 & $0.40-2.24$ \\
\hline & & 0.438 & АТСТССА & 0.016 & 0.57 & $0.14-2.36$ \\
\hline & & 0.262 & ATCTCGA & 0.014 & 0.31 & $0.04-2.41$ \\
\hline & & 0.227 & ATTTGCA & 0.012 & 2.92 & $0.51-16.59$ \\
\hline & & 0.576 & Rare & 0.010 & 1.61 & $0.31-8.44$ \\
\hline & & Male $\geq 90$ & СTCTGCA & 0.519 & 1.00 & Reference \\
\hline & & 0.787 & ATTTGCA & 0.014 & 1.36 & $0.15-12.76$ \\
\hline & & 0.411 & ATCTCGA & 0.024 & 0.42 & $0.05-3.29$ \\
\hline & & 0.000 & АТСТССА & 0.012 & 0.00 & - \\
\hline & & 0.109 & ATCTGCA & 0.053 & 2.52 & $0.81-7.82$ \\
\hline & & 0.181 & ATCCGCA & 0.123 & 1.63 & $0.80-3.35$ \\
\hline & & 0.390 & CTTTGCA & 0.105 & 1.46 & $0.62-3.43$ \\
\hline & & 0.150 & CTCCGGG & 0.028 & 2.48 & $0.72-8.56$ \\
\hline & & 0.004 & CTCCGCA & 0.040 & 6.20 & $1.80-21.30$ \\
\hline & & 0.518 & CCCTGCA & 0.076 & 1.38 & $0.52-3.67$ \\
\hline & & 0.516 & Rare & 0.007 & 2.45 & $0.16-36.74$ \\
\hline
\end{tabular}

${ }^{a}$ Number of tagSNPs among all SNPs genotyped for a candidate gene, ${ }^{b}$ The $P$ value for each haplotype using a general linear model (GLM).

lifespan in the GG haplotype of PAX4 (OR 5.14) among nonagenarian men. Although only the rs1980042 (C) allele in haplotypes of LYN seemed to confer longevity, the effect size of 2.5 for the $\mathrm{CC}$ genotype increased to 6.2 for the CTCCGCA haplotype among nonagenarian men. Among female 
A

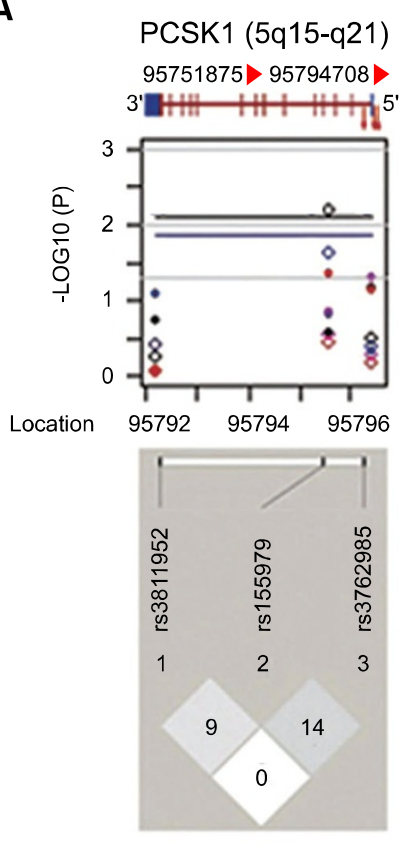

B
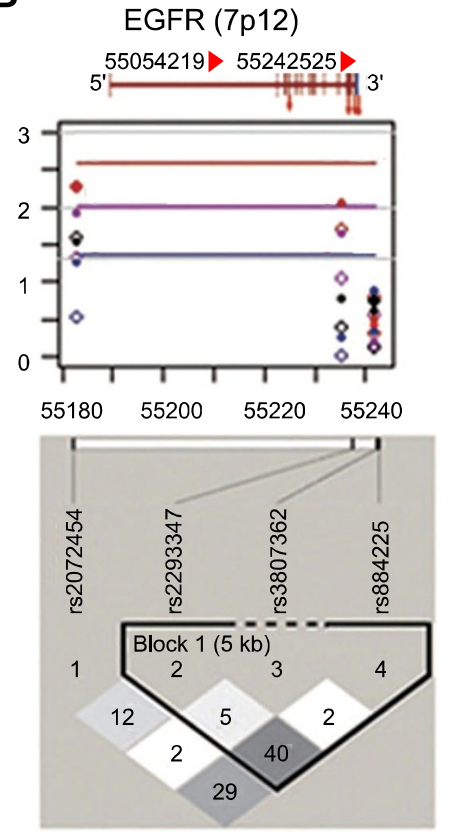

C
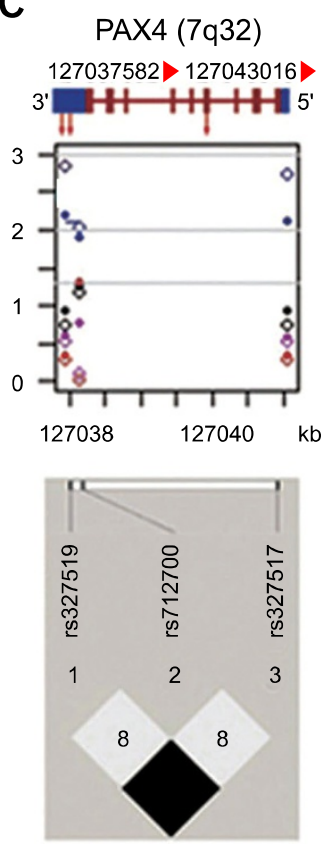

D
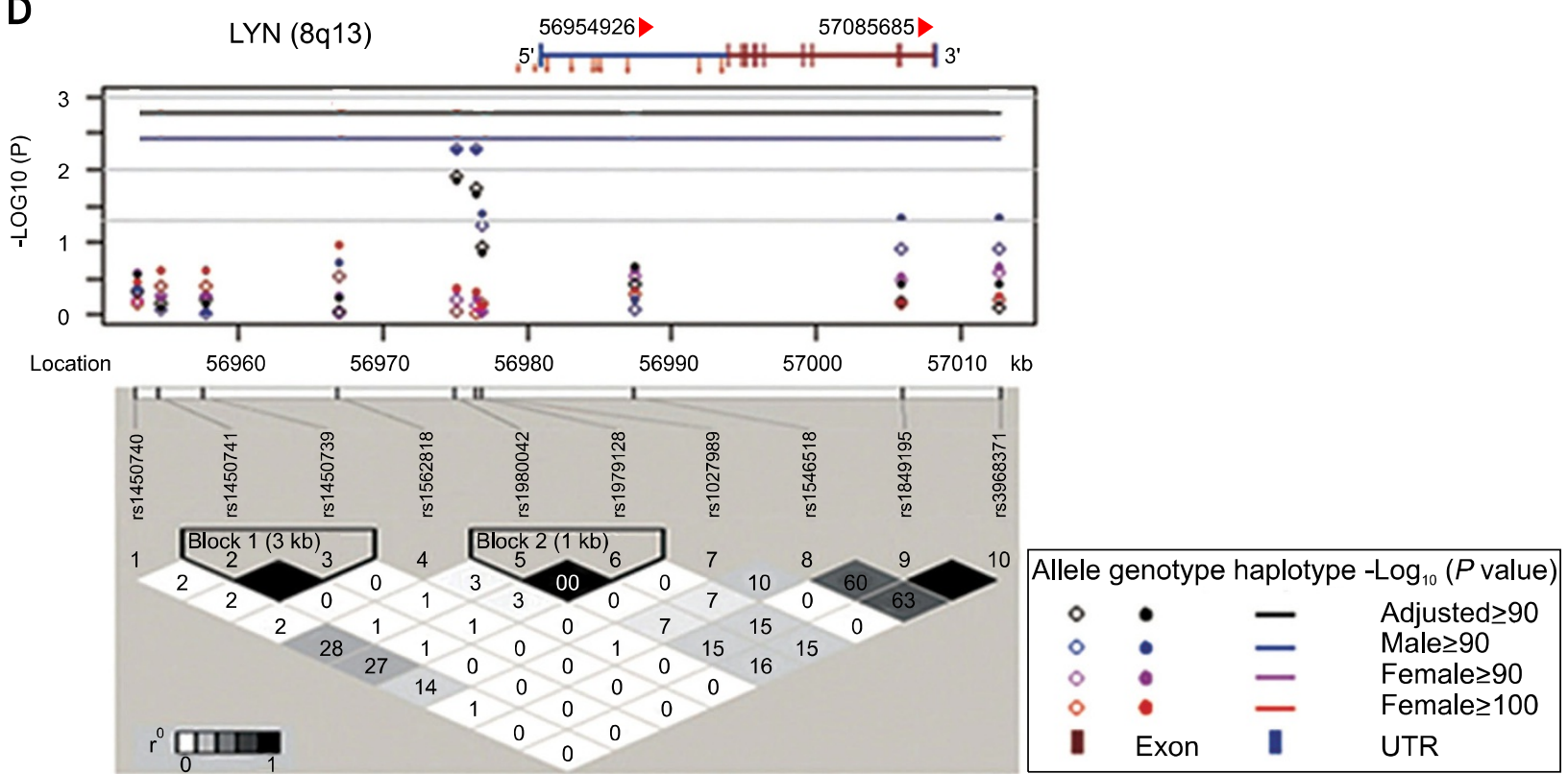

Figure 1. Summary results with gene and SNP information for four genes that consistently showed evidence for association with lifespan in allelic, genotypic, and haplotypic analyses.

centenarians, the OR of 3.12 for the rs2293347 (GG) genotype increased to 4.11 for the CGCA haplotype of the EGFR gene when compared with the CACA reference haplotype.

\section{Gene-gene interactions}

Supplement Data Table S2 presents significant $P$ values for the epistatic interaction LRT under a best fitting model among five genetic models tested for each age/gender group. Supplement Data Table S3 shows the ORs and their 95\% Cls for the genotype combinations composed of each candidate SNP pair for two genes along with the numbers of cases and controls. For each age/gender group, $6,1,3$, and 2 pairs of loci for each group 
yielded statistical significance in both the regression model and ORs for interaction. In particular, the interaction of platelet derived growth factor $C$ (PDGFC) and glycogen synthase 2 (liver) (GYS2) for males older than $90 \mathrm{yr}$ as well as the interaction of TNFSF11 and CDK10 in the group adjusted by gender showed a dose-response relationship with increasing copies of the target allele for each SNP.

\section{Discussion}

It is well known that genetic variation exists across ethnic groups. Indeed, significant association of genes for human leukocyte antigen (HLA), ACE, $A P O E, M T T P$, and CETP with human longevity in Caucasian populations have not been replicated in studies using Korean centenarians (Choi et al., 2003). The Korean longevity study carried out since 1998 reported significant differences in the occurrence of centenarians by gender, location, lifestyle, and diet (Lee et al., 2005). In 2005, the ratios of the Jeola province to the Kyongsang province were 1:0.97 for nonagenarians and 1:1.27 for centenarians (KNSO, 2005). Interestingly, a reverse relationship between the incidence of cancer and degree of longevity has been observed across provinces, indicating that prevention of cancers may be an important strategy to enhance lifespan (Kwon and Park, 2005). This notion could be applied to other age-related diseases such as DM and CVD.

According to the theory of evolution, differential survival reflects the effect of deleterious genotypes of age-related diseases. However, some longevity genes (e.g. klotho that is associated with low HDL) buffer the deleterious effect of age-related diseases genes (e.g. lipoprotein, Lp(a), a susceptibility gene for vascular disease), which may explain an observed paradoxical increase of deleterious genotypes in centenarians (Bergman et al., 2007). Lunetta et al. (2007) conducted a genome-wide association study of age-related phenotypes using Affymetrix $100 \mathrm{~K}$ SNP Chips in the context of a cross-sectional study design. However, none of the associations with 5 aging traits achieved genome-wide significance using 1,345 Framingham Study participants.

In the current study, a total of 60 genes out of 179 candidates were nominally associated $(P<$ $0.05)$ with longevity in at least one of age/gender groups. Among eleven genes that consistently yielded statistical evidence of allelic, genotypic and haplotypic association with lifespan, only four genes remained significant after the Bonferroni correction for haplotypes. The protein encoded by the PCSK1 gene, located in $5 q 15-q 21$, is a proinsulin-processing enzyme that plays a key role in regulating insulin biosynthesis (Ohagi et al., 1996). Mutations in this gene are associated with obesity, tumorigenesis, and metastasis (Tzimas et al., 2005). The PAX4 gene, located in $7 q 32$, is a member of the paired box family of transcription factor that mediates differentiation of insulin-producing beta-cells in the pancreas and plays critical roles during cancer growth (Li et al., 2006). EGFR is a transmembrane glycoprotein that functions as a tyrosine protein kinase (Kondo and Shimizu, 1983). Mutations in the EGFR gene, located in the $7 p 12.3-p 12.1$ region, have oncogenic potential in the development of non-small cell lung cancer (Tai et al., 2006). The $L Y N$, located on $8 q 13$, encodes a tyrosine kinase that is essential in immunoglobulin-mediated signaling, particularly in establishing B cell tolerance (Parravicini et al., 2002). Even aging mice with a Lyn up/up phenotype do not display hematologic malignancies, while $\mathrm{Lyn}^{-/-}$mice are more susceptible to tumorigenesis (Harder et al., 2001). To the best of our knowledge, this is the first reported evidence associating these genes with human longevity.

Several limitations of this study deserve mention. First, the influence of environmental factors could not be controlled in this study due to lack of information in the control group. Second, we could not evaluate the function of any SNP that was not a missense mutation but did have statistical evidence for association. Third, given the small number of centenarians, the statistical power may not be sufficient to significantly detect genes with weak effect, particularly, for the gene-gene interaction. There are further issues concerning the interpretation of the findings using the over-dominant model and the control group consisted of 12-14 yr old middle school students. To eliminate the impact of different substructure between the long-lived group and the young control group, the $P$ values were corrected by genomic control for each age-gender group. In the nonagenarian group, the Bonferroni threshold for $P<0.05$ with 463 SNPs was $1.08 \times 10^{-4}$ and the significance level was relaxed by 1000 permutations to $1.69 \times 10^{-4}, 1.56$ $\times 10^{-4}, 2.16 \times 10^{-4}, 1.50 \times 10^{-4}$, and $2.42 \times 10^{-4}$ under the co-dominant, dominant, recessive, over-dominant, and log-additive models, respectively. None of the SNPS achieved genome-wide significance. However, the underlying LD structure among the markers reduced the number of effectively independent tests, and so the genome-wide threshold of significance may be too stringent particularly in the context of candidate gene approach (Rao and Gu, 2001). 
For genetic variants with strong effects, relatively small sample size is sufficient to achieve statistical power to detect association. The results in this study warrant further replication studies. However, the expectation for replication of results may need to be relaxed in studies of extremely old subjects, who are rarely observed. Under such circumstances, additional information gathered from laboratory techniques, bioinformatics, and a priori insights of biological pathways could be used to provide plausibility for interpreting genetic association findings (Chanock et al., 2007). To increase statistical power of a test for gene by gene or gene by environment interaction, international collaboration is essential due to the rarity of centenarians. Continuing efforts may be necessary to find a functional variant in the associated region.

Nevertheless, the results from various statistical analyses consistently supported that the presence of the target allele of a potent SNP conferred a greater likelihood of longevity in Koreans. The effects of gender mediated genetic association with the probability of achieving longevity, as shown in previous studies (Franceschi et al., 2000) and the observed gender difference in our findings supports, in part, the gender-specific probability of achieving longevity. Interestingly, a few candidate gene variants with no or insignificant allelic effect revealed strong evidence for the allele with a large heterozygote effect on longevity (e.g. MGP, TNFSF11, and $A B C C 8$ ).

As far as we know, this is the first association study of longevity susceptibility genes adopting a high-throughput candidate gene approach in nonagenarians and centenarians who rarely appear in Korea. An understanding of the mechanisms underlying aging and longevity by discovering novel susceptibility genes may lay the foundations for prediction, prevention, and treatment of age-related diseases. Thus, the findings of the current study may provide a starting point to unravel genetic factors controlling longevity for future studies.

\section{Methods}

\section{Subjects}

We identified 137 long-lived individuals over $90 \mathrm{yr}$ old including 35 centenarians from the Korean Centenarian Study, which began in 1999 (Choi et al., 2003). We also selected 213 healthy middle school students from individuals who visited the Samsung Seoul Hospital for psychiatric tests in 2003 to be used for comparison. Eight control samples from a three-generation family were included to test genotype quality. After informed consent was obtained from all subjects, a clinician interviewed study subjects and drew a $10 \mathrm{ml}$ fasting venous blood sample in an EDTA tube. The institutional review board (IRB) of the Samsung medical center reviewed the research protocols and approved this study. The gender and age composition of study subjects were shown in Supplemental Data Table S1.

\section{Candidate genes and SNP selection}

Candidate genes, mostly for DM, CVD, and cancers, were selected based on searches for their functions in public databases (OMIM, Gene, etc.) and previous literature reviews. SNP markers within or near candidate genes were identified from the literature, the dbSNP database (http://www.ncbi.nlm.nih.gov/projects/SNP/), the HapMap database (http://www.hapmap.org/), and Vector NTI ${ }^{\circledR}$ software (Invitrogen, Inc., Carlsbad, CA). We included 565 informative single nucleotide polymorphism (SNP) markers with high design scores provided by Illumina, Inc. (San Diego, CA) and heterozygosity above 0.05 using 300 independent healthy Korean samples. Information for these 565 SNPs located in 194 genes is presented in Supplemental Data Table S2.

\section{DNA preparation and SNP genotyping}

Genomic DNA samples were prepared from peripheral blood using the QIAamp DNA Blood Maxi kit following the manufacturer's instructions (Qiagen, Inc., Valencia, CA). For each sample, an average concentration of DNA from three repeated measures was determined using a SpectraMax Plus 384 spectrophotometer (Molecular Devices, Inc., Sunnyvale, CA). A $250 \mathrm{ng}$ aliquot of DNA was genotyped for SNP markers using Golden-Gate chemistry on Sentrix ${ }^{\circledR}$ Array Matrices (Illumina, Inc.) at the Samsung Biomedical Research Institute. Genotypes showing significant statistical evidence for association with longevity were confirmed using an ABI PRISM 3100 sequencer (Applied Biosystems, Inc., Foster City, CA).

\section{Statistical analysis}

At each SNP, the genotyping call rate (CR > 95\%), minor allele frequency (MAF $>2 \%$ ), and deviation from Hardy-Weinberg equilibrium (HWE $P<0.0001$ ) were separately computed in the long-lived group and the young healthy group. Ancestral alleles for each SNP were determined using the dbSNP database. The SNPs-based whole genome association studies (SNPassoc) program implemented in the free downloadable statistical software environment R v. 2.6.2 (http://www.r-project.org) was used to perform the quality tests for genotypes (González et al., 2007). We performed preliminary analyses of linkage disequilibrium (LD) patterns using both $D^{\prime}$ and $r^{2}$ and then selected a set of tagging SNPs within a gene for the haplotype-based analysis using Haploview v. 4.0 (http:// www.broad.mit.edu/mpg/haploview/ index.php/).

We performed subsequent statistical analyses for each of four groups stratified by age and gender (nonagenarians, male nonagenarians, female nonagenarians, and female centenarians). We assessed allelic association with the $\chi^{2}$-tests for individual SNPs and calculated the allelic 
odds ratio (OR) and 95\% confidence interval $(\mathrm{Cl})$ in both adjusted and stratified analyses using publicly available subroutines 'genassoc' and 'gamenu' in the STATA/SE v. 9 software package (http://www.gene.cimr.cam.ac.uk/clayton/ software/stata/).

We performed genotypic association tests and logistic regression analyses implemented in the SNPassoc package in five different genetic models: codominant, dominant, recessive, over-dominant, and log-additive. To account for the impact of substructure or genetic relatedness among the subjects, we estimated the 'inflation factor $(\lambda)^{\prime}$ for each age-gender group and corrected $P$ values by genomic control (Devlin and Roeder, 1999).

We used general linear models for the regression of longevity on ambiguous haplotypes. Rare haplotypes with an allele frequency of less than $1 \%$ were combined in a group to be analyzed. We applied logistic regression models (LRMs) to perform two-way interaction analyses between two SNPs for each gene (Briollais et al., 2007). For each of four age/gender groups, we screened the most promising pairs of SNPs that yielded $P<0.01$ for the interaction likelihood ratio test (i.e. $\mathrm{LRT}_{i j}=-2$ ( $\log \mathrm{L}_{\text {full(i,j)- }}$ $\left.\log \mathrm{L}_{\text {additive(i,j) }}\right)$ under the five genetic models. Subsequently, we obtained the ORs and $95 \%$ Cls under the best fitting model for both marginal effects and interactions of the pair of SNPs.

We computed the statistical power of our sample sizes for each genotype using the web browser 'Genetic Power Calculator' (http://pngu.mgh.harvard.edu/ purcell/gpc/cc2. html) under an additive model with the assumption of disease allele frequencies of $0.3, D^{\prime}$ of 1 , and a longevity prevalence of $0.13 \%$ and $0.002 \%$ for the Korean nonagenarians and centenarians, respectively (Purcell et al., 2003).

\section{Supplemental data}

Supplemental Data include three tables and can be found with this article online at http://e-emm.or.kr/article/article files/SP-41-11-01.pdf.

\section{Acknowledgements}

We appreciate the technical support of our staffs: En Jin Han, Hanee Kwon and Yonghun Lim at the Center for Genome Research of the Samsung Biomedical Research Institute and also gratefully acknowledge the hard work of those involved in collecting samples. This work was supported by the Research Program of dual regulation mechanisms of aging and cancer from the Korea Science and Engineering Foundation (M1075604000107N560400110); the Korea Research Foundation for Health Science; and Hallym University Research Fund, 2009 (HRF-2009-000).

\section{References}

Atzmon G, Rincon M, Schechter CB, Shuldiner AR, Lipton $\mathrm{RB}$, Bergman A, Barzilai N. Lipoprotein genotype and conserved pathway for exceptional longevity in humans. PLoS Biol 2006;4:e113
Bergman A, Atzmon G, Ye K, MacCarthy T, Barzilai N. Buffering mechanisms in aging: a systems approach toward uncovering the genetic component of aging. PLoS Comput Biol 2007;3:e170

Briollais L, Wang Y, Rajendram I, Onay V, Shi E, Knight J, Ozcelik $\mathrm{H}$. Methodological issues in detecting gene-gene interactions in breast cancer susceptibility: a populationbased study in Ontario. BMC Med 2007;5:22

Capri M, Salvioli S, Sevini F, Valensin S, Celani L, Monti D, Pawelec G, De Benedictis G, Gonos ES, Franceschi C. The genetics of human longevity. Ann NY Acad Sci 2006; 1089:516-37

Chanock SJ, Manolio T, Boehnke M, Boerwinkle E, Hunter DJ, Thomas G., Hirschhorn JN, Abecasis G., Altshuler D, Bailey-Wilson JE, Brooks LD, Cardon LR, Daly M, Donnelly P, Fraumeni JF Jr, Freimer NB, Gerhard DS, Gunter C, Guttmacher AE, Guyer MS, Harris EL, Hoh J, Hoover R, Kong CA, Merikangas KR, Morton CC, Palmer LJ, Phimister EG., Rice JP, Roberts J, Rotimi C, Tucker MA, Vogan KJ, Wacholder S, Wijsman EM, Winn DM, Collins FS. Replicating genotype-phenotype associations. Nature 2007;447: $655-60$

Choi YH, Kim JH, Kim DK, Kim JW, Kim DK, Lee MS, Kim CH, Park SC. Distributions of ACE and APOE polymorphisms and their relations with dementia status in Korean centenarians. J Gerontol A Biol Sci Med Sci 2003;58: 227-31

Christensen K, Johnson TE, Vaupel JW. The quest for genetic determinants of human longevity: challenges and insights. Nat Rev Genet 2006;7:436-48

Devlin B, Roeder K. Genomic Control for Association Studies. Biometrics 1999;55:997-1004

Franceschi C, Motta L, Valensin S, Rapisarda R, Franzone A, Berardelli M, Motta M, Monti D, Bonafé M, Ferrucci L, Deiana L, Pes GM, Carru C, Desole MS, Barbi C, Sartoni G., Gemelli C, Lescai F, Olivieri F, Marchegiani F, Cardelli M, Cavallone L, Gueresi P, Cossarizza A, Troiano L, Pini G, Sansoni P, Passeri G, Lisa R, Spazzafumo L, Amadio L, Giunta S, Stecconi R, Morresi R, Viticchi C, Mattace R, De Benedictis G, Baggio G. Do men and women follow different trajectories to reach extreme longevity? Italian Multicenter Study on Centenarians (IMUSCE). Aging (Milano) 2000;12: 77-84

González JR, Armengol L, Solé X, Guinó E, Mercader JM, Estivill X, Moreno V. SNPassoc: an R package to perform whole genome association studies. Bioinformatics 2007;23: 644-5

Harder KW, Parsons LM, Armes J, Evans N, Kountouri N, Clark R, Quillici C, Grail D, Hodgson GS, Dunn AR, Hibbs ML. Gain- and loss-of-function Lyn mutant mice define a critical inhibitory role for Lyn in the myeloid lineage. Immunity 2001;15:603-15

Jee SH, Ohrr H, Sull JW, Yun JE, Ji M, Samet JM. Fasting serum glucose level and cancer risk in Korean men and women. JAMA 2005;293:194-202

Kondo I, Shimizu N. Mapping of the human gene for epidermal growth factor receptor (EGFR) on the p13 leads to q22 region of chromosome 7. Cytogenet Cell Genet 


\section{3;35:9-14}

Korea National Statistical Office: Statistical Annual Report 2005. Deajeon, Republic of Korea

Kwon IS, Park SC. Gender-Specific and Age-Dependent Changes in Health Status and Medical Characteristics of Korean Centenarians. Kor J Genrontol 2005;15:10-25

Lee MS, Yeo EJ, Kwak CS, Kim K, Choi YH, Kwon IS, Kim $\mathrm{CH}$, Park SC. Gender differences in health and nutritional status of Korean centenarians. Kor J Gerontol 2005;15: 65-75

Li Y, Nagai H, Ohno T, Ohashi H, Murohara T, Saito H, Kinoshita T. Aberrant DNA demethylation in promoter region and aberrant expression of mRNA of PAX4 gene in hematologic malignancies. Leuk Res 2006;30:1547-53

Lunetta KL, D'Agostino RB Sr, Karasik D, Benjamin EJ, Guo CY, Govindaraju R, Kiel DP, Kelly-Hayes M, Massaro JM, Pencina MJ, Seshadri S, Murabito JM. Genetic correlates of longevity and selected age-related phenotypes: a genomewide association study in the Framingham Study. BMC Med Genet 2007;8(Suppl 1):S13

Mackness M, Mackness B. Paraoxonase 1 and atherosclerosis: is the gene or the protein more important? Free Radic Biol Med 2004;37:1317-23

McGue M, Vaupel JW, Holm N, Harvald B. Longevity is moderately heritable in a sample of Danish twins born 1870-1880. J Gerontol 1993;48:B237-44

Ohagi S, Sakaguchi H, Sanke T, Tatsuta H, Hanabusa T, Nanjo K. Human prohormone convertase 3 gene: exon-intron organization and molecular scanning for mutations in Japanese subjects with NIDDM. Diabetes 1996;45:897-901

Parravicini V, Gadina M, Kovarova M, Odom S, GonzalezEspinosa C, Furumoto Y, Saitoh S, Samelson LE, O'Shea JJ, Rivera J. Fyn kinase initiates complementary signals required for IgE-dependent mast cell degranulation. Nature
Immun 2002:3:741-8

Perls TT, Bubrick E, Wager CG, Vijg J, Kruglyak L. Siblings of centenarians live longer. Lancet 1998;351:1560

Puca AA, Daly MJ, Brewster SJ, Matise TC, Barrett J, Shea-Drinkwater M, Kang S, Joyce E, Nicoli J, Benson E, Kunkel LM, Perls T. A genome-wide scan for linkage to human exceptional longevity identifies a locus on chromosome 4. Proc Natl Acad Sci USA 2001;98:10505-8

Purcell S, Cherny SS, Sham PC. Genetic Power Calculator: design of linkage and association genetic mapping studies of complex traits. Bioinformatics 2003;19:149-50

Rao DC, Gu C. False positives and false negatives in genome scans. Adv Genet 2001;42:487-98

Rousseau MC, Parent ME, Pollak MN, Siemiatycki J. Diabetes mellitus and cancer risk in a population-based case-control study among men from Montreal, Canada. Int J Cancer 2006; 118:2105-9

Salvioli S, Olivieri F, Marchegiani F, Cardelli M, Santoro A, Bellavista E, Mishto M, Invidia L, Capri M, Valensin S, Sevin F, Cevenini E, Celani L, Lescai F, Gonos E, Caruso C, Paolisso G, De Benedictis G, Monti D, Franceschi C. Genes, ageing and longevity in humans: problems, advantages and perspectives. Free Radic Res 2006;40:1303-23

Tai AL, Sham JS, Xie D, Fang Y, Wu YL, Hu L, Deng W, Tsao GS, Qiao GB, Cheung AL, Guan XY. Co-overexpression of fibroblast growth factor 3 and epidermal growth factor receptor is correlated with the development of nonsmall cell lung carcinoma. Cancer 2006;106:146-55

Tzimas GN, Chevet E, Jenna S, Nguyên DT, Khatib AM, Marcus V, Zhang Y, Chrétien M, Seidah N, Metrakos P. Abnormal expression and processing of the proprotein convertases PC1 and PC2 in human colorectal liver metastases. BMC Cancer 2005;5:149 